\title{
Inside medical journals
}

\author{
Harvard Medical School - Boston, MA, USA
}

Trustworthy information about advances in medical care is made available in its most complete and balanced form in peer reviewed medical journals. The journals are respected but poorly understood. Both authors and readers have a stake in understanding how peer review and editorial decisions are done so that they can make more effective use of jornals.

Suzanne Fletcher and I have experienced journals as authors and readers, as editors of Annals of Internal Medicine, through our research on editing practices, and by many discussions with other editors. What follows is generally in common with peer reviewed journals throughout the world, though specific practices vary from journal to journal.

The purpose of peer review and editing is to select manuscripts for publication and to improve the completeness, balance and clarity of the presentation. Review is not an effective way of assuring the accuracy of the data. The time and effort in peer review, beyond what would be necessary to just publish the manuscript as received, is justified by the possibility of making the results more useful and so reducing the time from research idea to use of the results of research in practice.

Manuscripts submitted to journals are sent to external reviewers - typically two in academic positions who spend an average of three hours reviewing the manuscript and are not paid for this work. Some manuscripts, with little chance of success, are rejected without review, to save authors' and reviewers' time. Reviewers are chosen to have complementary expertise - for example, one in content area (such as congestive heart failure or amoebiasis) and the other in the methods (such as randomized trials or case-control studies). Reviewers and editors judge manuscripts on several dimensions: importance of the question, match with the readers' interests, scientific strength of the methods, and quality of the writing. Reviewers are asked to provide confidential comments for and extensive suggestions for the authors. The acceptance rate depends on the number of manuscripts received and the space available in the journal. For large, highly competitive journals acceptance is 15 $20 \%$; for the majority of the world's journals, with circulation under 5,000, it is higher. Reviewers commonly do not agree on their recommendations for or against acceptance, which might be expected since they have different perspective from each other and from the editors. Final decisions are judgements made by editors and their staff, not the result of counting reviewers' votes. The initial reviews take 10 or more weeks and usually lead to suggestions for improvement whether or not the manuscript is accepted.

How effective is peer review and editing? Most editorial practices are supported by tradition and argument, not research. In a series of studies of peer review and editing, we have assessed some common practices. We showed, in a randomized controlled trial, that "blinding" reviewers to authors' names and institutions improves the quality of reviews. Reviewers tended to be better is they are early in their career, an observation that is supported by the only other published study of this question and is contrary to conventional wisdom. In a study of how the quality of reporting changes from receipt of manuscripts to publication, we showed that nearly all aspects of reporting improve, especially discussion of limitations and generalizability and the balance of conclusions. Reading level for published articles was difficult (about grade 16); it was improved a little by review and editing. 